\title{
Parental non-concordance with occlusion therapy
}

\author{
David Newsham
}

\begin{abstract}
Backgroundlaims-Non-concordance has often been reported as a major contributor to the failure of occlusion therapy for amblyopia. In other fields of medicine the extent of a patient's understanding in areas of the disease and treatment has been shown to have both a direct and indirect effect on subsequent concordance. The aims of this study were to determine the extent of parental non-concordance, to assess their level of understanding in key areas of amblyopia, occlusion therapy, critical period and prognosis, and to discover the parent's own reasons for failing to concord.
\end{abstract}

Methods-Parents of children aged 2-7 years receiving a minimum of 1 hour of occlusion for unilateral amblyopia were recruited. Parental concordance was monitored using a diary and their understanding and reasons for nonconcordance were assessed by a questionnaire. Concordance was analysed by calculating a concordance index, determining the proportion of nonconcordance, and also by classifying the non-concordance on the basis of whether the behaviour was intentional or unintentional and whether the parents were adequately or inadequately informed.

Results-Parental non-concordance was defined as failing to occlude less than $\mathbf{8 0 \%}$ of the total prescribed time. The median concordance index was 0.75 and the proportion of non-concordant parents was $0.54(95 \% \mathrm{CI} 0.41$ to 0.67$)(\mathrm{n}=57)$. Parental knowledge was poor in areas of the critical period with $23 \%$ of parents unaware of an age limit to the treatment. Reasons for non-concordance given by $68 \%$ of parents demonstrated poor knowledge.

Conclusion-A substantial proportion of the non-concordant parents had poor understanding in areas such as the critical period and errors also occurred in implementing the treatment regimen. Increased parental awareness of the rationale and urgency of the treatment, with reinforcement of details of the regimen, would help to reduce nonconcordance with occlusion therapy.

(Br F Ophthalmol 2000;84:957-962)

Liverpool, Thompson

Yates Building,

Liverpool L69 3GB,

UK

D Newsham

Correspondence to: $\mathrm{Mr} \mathrm{D}$

Newsham

newts@liv.ac.uk

Accepted 26 April 2000 tor that determines whether or not occlusion is successful. ${ }^{1-5}$ Few studies, however, have pri-

Non-concordance affects many areas of medicine and the use of occlusion therapy for amblyopia is no exception. Several studies have reported that concordance is an important fac- marily investigated non-concordance and, of those that have, most have disadvantages such as recording concordance only if $100 \%$ clinic attendance is achieved, retrospective designs, or use of private patients with unusually close monitoring. ${ }^{467}$ As non-concordance is a major barrier to the successful use of occlusion, it is important to determine the factors that may contribute to poor concordance. There appears to be disagreement as to whether non-concordance decreases with age, ${ }^{4}$ increases with age, ${ }^{5}$ or is not related to age, ${ }^{6}$ but it seems likely that there is a positive correlation with social deprivation. ${ }^{6}$ It is also uncertain whether the visual acuity at the start of treatment has an effect on non-concordance, with reports of lower visual acuity leading to poor concordance, ${ }^{4}$ but others finding no such relation. ${ }^{6}$

Research in other areas of medicine has shown that understanding by the patient in key areas of the disease and treatment has a significant effect on the level of concordance ${ }^{8}$ and is illustrated by Ley's cognitive model ${ }^{9}$ (Fig 1). Understanding has a direct effect on memory, satisfaction, and concordance and, through its effect on memory and satisfaction, it also has an additional indirect effect on concordance. In other areas of medicine the patient's knowledge of the treatment regimen and understanding of what the doctor had said were shown to be significantly and independently related to concordance..$^{10}$ Poor understanding by parents has also been shown to account for their poor concordance in implementing asthma medicines. ${ }^{11}$ This is a similar situation to use of occlusion therapy where the extent of the parent's knowledge is important as they are responsible for the implementation of the treatment on their child. It is crucial therefore that the parents are fully aware of all aspects of amblyopia, such as the critical period and the consequences of not occluding their child. In other areas of medicine the subjective perception of the severity of an illness by mothers of paediatric patients has been shown to be an important factor affecting concordance. ${ }^{12}{ }^{13} \mathrm{It}$ seems logical that concordance would be more likely to be achieved if those responsible for implementing the treatment are fully aware of their child's defect, its severity, how they can improve the vision by occlusion, the lack of alternatives, and the urgency of the treatment.

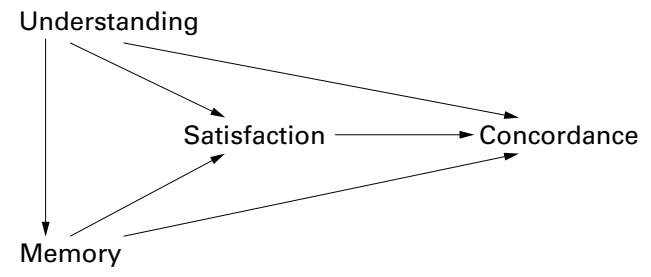

Figure 1 Ley's cognitive model. 
Indeed, understanding of a disease and treatment and the method of information exchange are thought to be vital factors which need to be taken into consideration when trying to improve concordance. ${ }^{14}$

Parental understanding in the use of occlusion for amblyopia involves three main areas: (1) parental knowledge of the details of the treatment regimen; (2) parental understanding of the rationale of occlusion; and (3) parental understanding of amblyopia and the critical period. Deficiency in the first area will obviously cause non-concordance-for example, not occluding for the correct number of hours per day or occluding the incorrect eye. Deficiency in the second area may result in parents incorrectly believing the occlusion will correct the strabismus (as the deviating eye straightens to fix while the occlusion is worn), causing disillusionment when this does not happen. This example indicates how parents could perceive occlusion as being ineffective due to a misunderstanding of the role of occlusion. Also, parents may incorrectly believe that surgery is an alternative to improve the acuity if the occlusion fails. Deficiency in the third area could cause non-concordance because of failure to appreciate the severity of the condition or the urgency resulting from the critical period. The influence of the level of parental understanding on concordance does not appear to have been investigated in the field of occlusion therapy. The aims of this study were to determine the level of parental non-concordance, to assess the level of parental knowledge regarding amblyopia, prognosis and occlusion, and to discover parents' own reasons for non-concordance.

\section{Methods}

All patients (new and existing) based on the inclusion criteria of receiving a minimum of 1 hour's occlusion for all types of unilateral amblyopia were eligible for recruitment into the study following informed consent from their parents. The written information document about the study that was given to the par-

Table 1 Parental awareness of amblyopia and knowledge of occlusion, critical period, prognosis, and treatment regimen

\begin{tabular}{ll}
\hline Questions & $\begin{array}{l}\text { No (\%) of parents giving } \\
\text { the correct answer }(n=57)\end{array}$ \\
\hline $\begin{array}{l}\text { Does your child have reduced vision in one eye? } \\
\text { What is the treatment for reduced vision in one eye? }\end{array}$ & $56(98)$ \\
$\begin{array}{l}\text { Does the treatment have to be carried out by a certain age? } \\
\text { Would it be easier to correct weak vision in one eye at 2 years or } 6\end{array}$ & $44(77)$ \\
$\begin{array}{l}\text { years of age? } \\
\text { Which eye are you putting the patch on? }\end{array}$ & $48(84)$ \\
How many hours were you told to put the patch on for? & $54(95)$ \\
\hline
\end{tabular}

Table 2 Parental reasons for non-concordance.

\begin{tabular}{ll}
\hline Reasons indicated by parents for their non-concordance & No (\%) of parents $(n=31)$ \\
\hline My child has a strong dislike to wearing the patch & $6(19)$ \\
I do not like the appearance of the patch on my child & $0(0)$ \\
I sometimes forget to put the patch on & $3(10)$ \\
Poor cooperation of the child & $0(0)$ \\
Social occasions or family activities & $1(3)$ \\
I will wait until my child is older and more cooperative & $14(45)$ \\
The treatment does not work & $5(16)$ \\
I will wait until my child understands the reasons for the treatment* & $2(7)$ \\
\hline
\end{tabular}

^Other reasons given by parents in preference to the alternatives provided in the questionnaire. ents was worded so as to be non-judgmental, indicating that the aim was to assess difficulties experienced by parents when occluding their child. Patients were not included or excluded on the basis of visual acuity. Each patient who visited the orthoptic department and fulfilled the inclusion criteria during a 3-4 month recruitment period was invited to be included in the study. Concordance was monitored by means of a diary in which parents were asked to record the length of time the patch was worn (to the nearest half hour) each day for a 4 week period. These instructions were typed at the top of the diary, which consisted of a table of 28 cells numbered day 1 to day 28 and in each cell the text "number of hours" was typed with a space where the parent was to record how long the patch was worn. Parental understanding and knowledge of amblyopia, occlusion therapy, critical period and prognosis, along with reasons for non-concordance were assessed by means of a questionnaire. To determine reasons for non-concordance, parents were given the option of choosing from six alternatives (commonly experienced by the author) or, if preferred, they could state their own alternatives. It was indicated on the questionnaire that more than one reason could be given if the parent wished. Content and face validity of the questionnaires was assessed by several orthoptists. This was achieved by gaining advice on the alternatives listed and questions to be included in the questionnaire from professionals regularly in contact with parents of amblyopic children. No revisions were deemed necessary but it was suggested that a reading ease score (Flesch Reading Ease) should be calculated using Microsoft Word to ensure the document was easily readable. A score of 85 on a 100 point scale was obtained which was well above the recommended minimum score of 70 . The diaries and questionnaires were also piloted on parents of 20 children receiving occlusion. Following this, an extra question ("Would occlusion be more effective at 2 years or 6 years?") was included to ascertain whether parents were aware that the prognosis may reduce as the child grows older. Details of the six questions used to assess parental knowledge and the six possible alternatives (or other given by the parent) that were listed in the questionnaire for nonconcordance are given, together with the scores obtained, in Tables 1 and 2 .

The diaries and questionnaires were given to the parent(s) who were with the child while at the orthoptic clinic, immediately after the parent had agreed to take part in the study and informed consent had been obtained. The diaries were collected when the patient and parent returned to the clinic after the 4 week period at which time the questionnaires were completed and collected to maximise return rates. It was made clear to the parents that the completed questionnaires and diaries would not be seen by the orthoptist responsible for their child's care. This was achieved by issuing the parents with an envelope in which to enclose the completed documents and an option to post the documents directly to the author if they so 


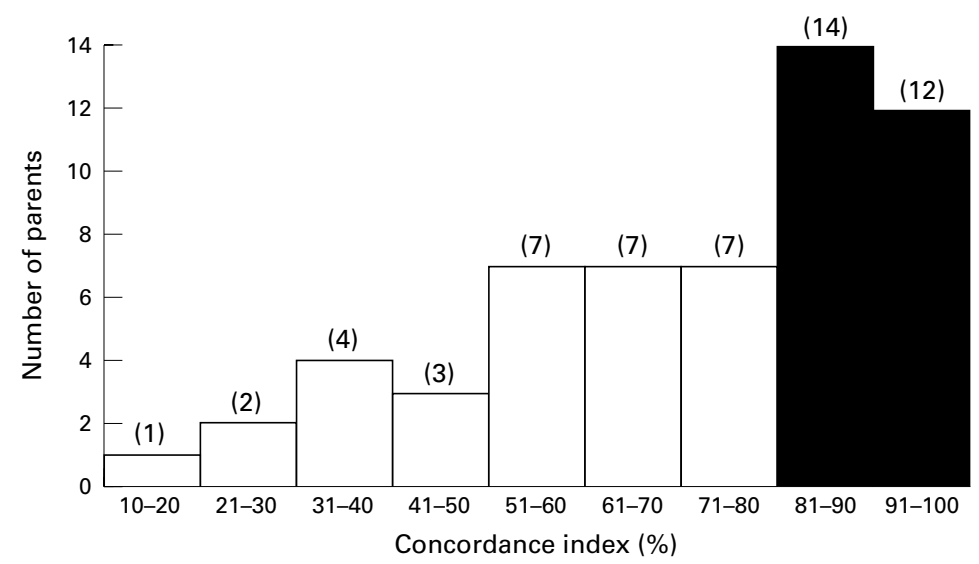

Figure 2 Distribution of parents in relation to the concordance index $(n=57)$. Open boxes represent non-concordant parents $(n=31)$; solid boxes represent concordant parents $(n=26)$.

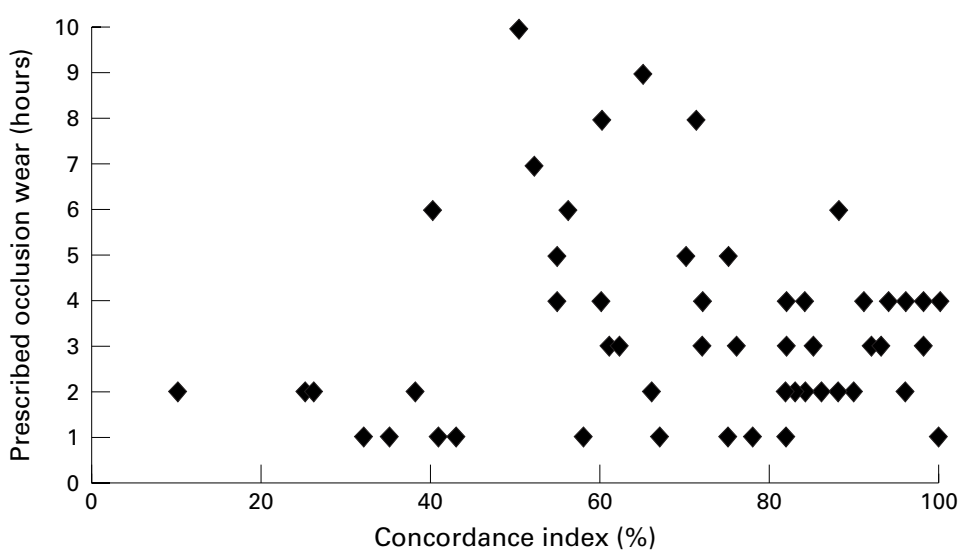

Figure 3 Correlation between the concordance index and level of prescribed occlusion ( $n=$ 57). $r=-0.02 ; p>0.10$ (Pearson product moment correlation test).

wished. These measures were taken to encourage the parents to record the level of concordance honestly without any fear of recompense.

Concordance was analysed by means of a concordance index ${ }^{15}$ calculated from occlusion recorded in the diary (hours)/prescribed occlusion (hours). The number of hours refers to the total number of hours for which the occlusion was worn as recorded in the diary over the 4 week period and the total amount of prescribed hours over the 4 week period, respectively. The concordance index provides a useful assessment of the proportion of prescribed occlusion that was achieved by the parents over a given period. It does not, however, give any information about how long the occlusion was performed on a day to day basis and hence whether the occlusion was evenly distributed throughout the duration of the treatment period. An alternative assessment of non-concordance is to calculate the proportion of non-concordant parents, which requires a threshold of concordance to be specified. For the purposes of this study a level of more than $80 \%$ of the total prescribed occlusion wear over the 4 week period was considered as concordant behaviour. The reasons for the nonconcordance based on the results of the level of parental understanding and reasons given by the parents themselves were analysed using Ley's classification of non-concordance. ${ }^{16}$ This involves determining, firstly, whether the nonconcordance was intentional or unintentional and, secondly, whether the parents were adequately or inadequately informed about amblyopia, occlusion, and the critical period. Four groups of behaviour were therefore formed: (1) adequately informed intentional non-concordance; (2) inadequately informed intentional non-concordance; (3) adequately informed unintentional non-concordance; and (4) inadequately informed unintentional nonconcordance.

\section{Results}

\section{CONCORDANCE}

No parents refused to take part in the study but, of the 65 patients recruited, eight were excluded because of missing data (diaries lost or not returned because of failure to attend appointments) leaving 57 patients for analysis. The median, $25 \%$, and $75 \%$ quartiles of age were 5,3 , and 6 years (range $2-7$ years). The median, $25 \%$, and $75 \%$ quartiles of length of prescribed occlusion were 3,2, and 4 hours (range 1-10 hours), with a range of amblyopia of strabismic, anisometropic, or strabismic and anisometropic combined. The age or length of prescribed occlusion of the excluded patients did not differ markedly from the remaining sample. The median concordance index was 0.75 with $25 \%$ and $75 \%$ quartiles of 0.57 and 0.88 , and the proportion of non-concordant parents was 0.54 (95\% confidence interval 0.41 to 0.67 ) (Fig 2).

Concordance indices were plotted against occlusion wear to determine whether a linear relation (negative correlation) was present, with greater lengths of prescribed occlusion wear resulting in less concordance (Fig 3 ). The correlation between the concordance index and length of prescribed occlusion wear was not significant $(r=-0.02, \mathrm{p}>0.10)$.

\section{PARENTAL KNOWLEDGE}

The questionnaires revealed that parental understanding was poor in important areas of the critical period such as knowledge of the age limit at which occlusion can still be effectively used and knowledge of the reducing prognosis with age. Errors also occurred in implementing the treatment regimen (Table 1). Reasons given by the parents for their non-concordance were varied (Table 2). No parent provided more than one reason, but a reason for non-concordance was indicated by each of the parents with a non-concordance index of 0.80 or less. Data are only provided for those parents who were classified as non-concordant. Only two parents with a concordance index of more than 0.80 provided reasons. As these parents were classified as concordant for the purposes of this study, and as the nonconcordance was minimal, it was not considered appropriate to include their reasons for non-concordance. The most common reasons given by $68 \%$ of the non-concordant parents indicated poor knowledge - for example, "I will wait until my child is older and more cooperative", "I will wait until the child 
understands the reasons for the treatment", "The treatment does not work".

NON-CONCORDANT BEHAVIOUR

Parental non-concordance was analysed to assess whether the behaviour was intentional or unintentional and whether the parents were adequately informed or inadequately informed. Of the 31 non-concordant parents, seven $(22 \%)$ indicated adequately informed intentional non-concordance. This was determined by the parents' reasons for the nonconcordance such as social occasions or because the child disliked the patch. Understanding and knowledge in these parents was complete. Inadequately informed intentional non-concordance was indicated by 18 parents $(58 \%)$. This was demonstrated by incomplete parental knowledge of the critical period in addition to reasons indicating intentional nonconcordance (Table 2). Adequately informed unintentional non-concordance was indicated by three parents $(10 \%)$ and was demonstrated by parents who reported that they sometimes forgot to put the patch on, yet had complete knowledge and understanding. Finally, inadequately informed unintentional nonconcordance was indicated by three parents $(10 \%)$ and was demonstrated by poor knowledge, and hence errors in implementing the treatment regimen. These parents were unknowingly occluding the incorrect eye as a result of problems with the information exchange between the orthoptist and parent.

\section{Discussion}

The extent of parental non-concordance was substantial with the proportion of nonconcordant parents at $54 \%$. This is similar to the level found by Smith et $a l^{6}$ (49\%), although the measure of concordance $(100 \%$ clinic attendance) is not comparable. The level in this study is higher than that of Nucci et $a l^{4}$ $(37-12 \%)$ and Lithander and Sjostrand $(18 \%)$, but this may be attributable to methodological differences such as retrospective designs and the use of private patients with close monitoring, respectively. The population recruited in this study was considered to be representative of the general amblyopic population with the exception that no patients with stimulus deprivation amblyopia were actually included. This occurred because no patients with this type of amblyopia presented during the recruitment period and reflects the fact that this type of amblyopia is less common. There would seem to be no reason to expect understanding to differ between this group and the study population. It is also unlikely that concordance in this group would markedly differ from the rest of the study population. This is because, although patients with stimulus deprivation amblyopia may be expected to be prescribed longer amounts of occlusion in line with the expected severity of the amblyopia, no correlation between this variable and concordance was found in this study.

Analysis using the concordance index could be criticised as it does not provide data on whether the occlusion was distributed evenly throughout the treatment period. However, as little is known about the dose-effect response of occlusion, an overall indication of the level of concordance was considered to be satisfactory. It is also accepted that the decision of where to insert the threshold of concordance will affect the proportion of non-concordant parents. The $80 \%$ threshold was adopted following the pilot study, as there appeared to be a dividing line between the distribution of the concordance indices at this point. Also, it was considered that, if concordance falls below $80 \%$ of the treatment time, a substantial amount of the treatment would then start to be omitted.

It is acknowledged that the true level of nonconcordance in this study may be higher as patients were excluded because of failing to attend or failing to return the diaries. It is possible that parents who showed nonconcordance with the study or non-attendance would have a greater level of non-concordance than the rest of the sample. Use of the diary method to monitor concordance may also have resulted in underestimation of the level of nonconcordance. This may arise in some cases because parents may be reluctant to disclose the full extent of their non-concordance. Assuring confidentiality may have helped to overcome this disadvantage to some degree. It should be noted, however, that all methods of measuring concordance have some weaknesses. It has been acknowledged that even the results from objective occlusion dose monitors (whilst not thought to be a likely scenario) could be distorted, for example, by placing the monitor on the arm. ${ }^{15}$ If a parent knows that the information given in the diary will not be seen by the orthoptist treating their child, there would appear to be no reason for them to be dishonest when disclosing the level of concordance. In other areas of medicine parental reports have been shown to compare well with objective techniques, ${ }^{17}$ and if bias is unavoidable it is desirable to underestimate the level of non-concordance rather than risk reporting a problem which may not actually exist.

Many patients benefit from full time occlusion. This study found no sign of any correlation between increased levels of prescribed occlusion and reduced concordance, with no evidence of a pattern or direction to the points (Fig 3), although it is acknowledged that the data were primarily investigated for a linear relation. Therefore, despite the extent of parental non-concordance, this would indicate that orthoptists should not be reluctant to prescribe larger amounts of occlusion out of concern that it will inevitably lead to greater nonconcordance. No other studies have investigated this correlation, but it has been reported that the level of visual acuity is not correlated with the degree of concordance. ${ }^{6}$ This is a similar finding as, generally speaking, the greater the severity of the amblyopia the longer the occlusion is prescribed.

Parental knowledge of the end of the critical period and reducing prognosis with age was poor. It is therefore not surprising that, in a large proportion of the non-concordant 
parents (68\%), the reason for their nonconcordance was related to poor knowledge and understanding. These parents did not perceive any urgency to the treatment because they were unaware of the critical period. In other words, the parents were unaware of their child's vulnerability to the consequences of not implementing early treatment of amblyopia, which can often lead to non-concordance. In general medicine a major study in the area of concordance was undertaken to explore relation between concordance and the patient, the treatment regimen, and the interaction between the physician and patient. ${ }^{18}$ The most common finding was that these factors showed no association with concordance. However, there were exceptions-for example, the influence of family and friends, concordance with other aspects of the regimen, duration of treatment, complexity of the regimen, patient's satisfaction, patient's expectations being met, the level of supervision by the clinician, and the Health Belief Model variables. The Health Belief Model variables ${ }^{19}$ are the patients' perceptions of their vulnerability, the seriousness of their illness, and the efficacy of the treatment which may affect their decision of whether or not to concord. The results of the present study indicated that parents were often unaware of their child's vulnerability to amblyopia and, with respect to efficacy of treatment, some parents indicated that they did not concord as they felt "the treatment did not work". Both of the variables from the Health Belief Model that have been shown to contribute to non-concordance were found in this study and can be attributed to poor understanding. These key studies conducted in general medicine into non-concordance show that understanding can have a substantial effect on concordance and there is no reason to expect this to be different for the treatment of amblyopia.

It could be argued that parents who believe the treatment is ineffective do not, in fact, have poor knowledge but are justified given the recent discussions concerning screening and the lack of experimental evidence regarding the efficacy of occlusion treatment. It is impossible to know how many parents would be aware of the recent criticisms of the lack of controlled trials demonstrating the efficacy of occlusion or research into the natural history of amblyopia. However, parents are just as likely to be aware of the large amount of anecdotal evidence supporting the use of occlusion as of the lack of trials. Some parents may incorrectly believe that occlusion is ineffective because of a misconception that it contributes to the alignment of a strabismus but, unfortunately, the reason why some parents in this study thought the treatment would be ineffective is not known. With these reservations, the relatively small proportion of non-concordant parents $(16 \%)$ who indicated that the treatment is ineffective have been included as having poor knowledge. Most of the parents $(52 \%)$ classed as demonstrating poor knowledge were unaware of the critical period. Some of the other factors that have been shown to be associated with non-concordance, such as patient's expectations being met and patient's satisfaction, are also related to levels of understanding. The extent to which a parent understands the issues surrounding the prognosis, amblyopia and treatment may therefore determine whether or not they feel the benefits of concordance outweigh the disadvantages of having to enforce the treatment.

Three parents in this study were found to have made serious errors in correctly executing the treatment regimen. While this is only a small percentage of the sample $(5 \%)$, it is still of concern that these children were, in fact, having their amblyopic eye occluded. This may have arisen as a result of a misunderstanding between the orthoptist and the parent or perhaps because of poor memory on behalf of the parent. Again, both of these areas have connections with understanding which, as discussed earlier in Ley's cognitive model, has a direct effect on memory. It is acknowledged that other factors may have an impact on parental understanding such as prior experience of occlusion, socioeconomic status, and the level of education of the parents which were not assessed in this study and could be an area of future research. It may prove difficult, however, to quantify the extent to which experience of prior occlusion by parents many years ago contributes to the knowledge at the present time. It would also be difficult to ensure that those parents who were thought to have no previous experience of occlusion did not, in fact, have friends or more distant relatives whose children were receiving occlusion. It is for this reason that both new and existing patients were included in the study. While socioeconomic status was not documented in this study, previous research ${ }^{6}$ has found that this contributes to non-concordance and it is possible that this is in some part because of a lack of parental understanding of amblyopia and occlusion, although this is purely speculative.

The method used in this study to assess parental knowledge and beliefs was by means of a questionnaire. An alternative to this method would be to use more qualitative techniques such as interviews. The advantages of qualitative methods in providing depth of information can be outweighed, however, because of a lack of standardisation and introduction of bias from the interviewer. For this reason, questionnaires were used and, as a compromise, the parents were given the opportunity of including their own reason for non-concordance if they did not feel the choices provided on the questionnaire were appropriate.

Analysis of the parents' reasons for nonconcordance showed that $68 \%$ were inadequately informed. While it is of concern that this is such a large proportion, it is also encouraging that many of these parents may improve their concordance by such a simple technique of providing information. Further information and better understanding, of course, cannot be expected to improve concordance in all parents, as some will choose not to concord 
Table 3 Possible solutions to improve concordance for the different categories of non-concordant behaviour

\begin{tabular}{ll}
\hline Categories of non-concordance & Solution to improve concordance \\
\hline Adequately informed intentional & Increased supervision, use of occlusion at nursery or school \\
Inadequately informed intentional & Provision of written information \\
Adequately informed unintentional & Memory aids and reminders \\
Inadequately informed unintentional & Provision of written information \\
\hline
\end{tabular}

despite adequate knowledge. The nonconcordance in these parents needs to be addressed in other ways. Some parents may find it difficult to discipline their child, despite realising its importance. This may be overcome to some extent by occluding the child at school or nursery. It may also be possible in these cases, as indicated by Becker and Maiman, ${ }^{19}$ to improve the concordance by closer supervision. The possible effect of this may have already been inadvertently shown in occlusion therapy, ${ }^{7}$ with supervision every two weeks yielding a non-concordance level of only $18 \%$.

Non-concordance with occlusion therapy in this study was found to be substantial and has often been noted as a primary cause of treatment failure. It may be useful to screen for non-concordance either using the parental diary technique or with occlusion monitors as they become more readily available and cheaper to produce. Non-concordant parents could then be asked to complete a questionnaire to assess their knowledge and reasons for non-concordance with the treatment. This would allow analysis of their individual type of non-concordant behaviour and subsequently the most appropriate intervention to improve the concordance could be chosen (Table 3). It can be seen, therefore, that knowledge and understanding of amblyopia and issues surrounding the prognosis, critical period, and treatment regimen need to be improved in order to increase parental concordance and ensure that the treatment is correctly implemented. Parents cannot be expected to concord with occlusion treatment unless disillusionment is prevented by removing misconceptions about what occlusion can achieve, they are fully informed about the need for urgency to the treatment, and they completely understand the regimen that needs to be followed.

The author acknowledges advice and help from colleagues in undertaking the study at Southport and Ormskirk (NHS) Trust and St Helens Hospital/Community (NHS) Trust.

1 Beardsell R, Clarke S, Hill M. Outcome of occlusion treatment for amblyopia. If Pediatr Ophthalmol Strabismus 1999;36:19-24

2 Elder MJ. Occlusion therapy for strabismic amblyopia. Aust NZ F Ophthalmol 1994;22:187-91.

3 Noda S, Hayasaka S, Setogawa T. Occlusion therapy of Japanese children with anisometropic amblyopia without strabismus. Ann Ophthalmol 1993;25:145-7.

4 Nucci P, Alfarano R, Piantanida A, et al. Compliance in antiamblyopia occlusion therapy. Acta Ophthalmol Copenh 1992;70:128-31.

5 Oliver M, Neumann R, Chaimovich Y, et al. Compliance and results of treatment for amblyopia in children more than 8 years old. Am f Ophthalmol 1986;102:340-5.

6 Smith LK, Thompson JR, Woodruff G, et al. Factors affecting treatment compliance in amblyopia. $\mathcal{F}$ Pediatr Ophthaling treatment compliance in am
mol Strabismus 1995;32:98-101.

7 Lithander J, Sjostrand J. Anisometropic and strabismic amblyopia in the age group 2 years and above: a prospective tudy of the results of treatment. BrF Ophthalmol 1991;75: $111-6$.

8 Ley P. Cognitive variables and non-compliance. $f$ Compliance Health Care 1986;1:171-88.

9 Ley P. Understanding, memory, satisfaction and compliance. Br F Clin Psychol 1982;21:241-54.

10 Lorenc L, Branthwaite A. Are older adults less compliant with prescribed medication than younger adults? $\mathrm{Br} \mathcal{F}$ Clin Psychol 1993;32:485-92.

11 Fauroux B, Just J, Couvreur J, et al. Treatment understanding and compliance in asthma of children: results of a prospective survey. Rev Pneumol Clin 1992:48:8-12.

12 Gillum RF, Barsky A. Diagnosis and management of patient non-compliance. FAMA 1974;228:1563-7.

13 Human MT, Teglasi H. Parents satisfaction and compliance with recommendations following psychoeducational aswith recommendations following psychoeducational

14 Cameron R, Best JA. Promoting adherence to health behavior change interventions: recent finding from behavioral ior change interventions: recent finding from behav
research. Patient Educ Counselling 1987;10:139-54.

15 Fielder AR, Irwin M, Auld R, et al. Compliance in amblyopia therapy. Br f Ophthalmol 1995;79:585-9.

6 Ley P. The psychology of compliance. In: Oborne DJ, Gruneberg MM, Eiser JR, eds. Research in psychology and medicine. London: Academic Press, 979:2.

17 Rodewald LE, Pichichero ME. Compliance with antibiotic therapy: a comparison of deuterium oxide tracer, urine bioassay, bottle weights and parental reports. $\mathcal{F}$ Pediatr 1993;123:143-7.

18 Haynes RB, Taylor DW, Sackett DL, eds. Compliance in health care. Baltimore: Johns Hopkins University Press, 1979.

19 Becker MH, Maiman LA. Sociobehavioural determinants of compliance with health and medical care recommendations. MedCare 1975;13:10-24. 